\title{
The Effect of Medical Technology Innovations on Patient Outcomes, 1990-2015: Results of a Physician Survey
}

\author{
David E. Wamble, PhD; Michael Ciarametaro, MBA; and Robert Dubois, MD, PhD
}

\begin{abstract}
BACKGROUND: Developments in diagnostics, medical devices, procedures, and prescription drugs have increased life expectancy and quality of life after diagnosis for many diseases. Previous research has shown that, overall, increased investment in medical technology has led to increased health outcomes. In addition, the value of investment in specific innovations, particularly in new pharmaceuticals or biopharmaceuticals, has frequently been shown through an evaluation of the associated health outcomes and costs. Value assessments for all medical technologies and interventions are an important consideration in current debates on access and affordability of health care in the United States.
\end{abstract}

OBJECTIVE: To identify practicing physician impressions of the historical effect of postdiagnosis innovations in medical technology on patient outcomes within the 8 health conditions that have the largest effect on health in the United States.

METHODS: National statistics were used to identify the 8 conditions responsible for the most mortality and morbidity within the United States between 1990 and 2014. A physician survey was developed for each major condition to obtain physician opinion on the extent to which pharmaceuticals and biopharmaceuticals, medical devices, diagnostics, and surgical procedures contributed to improvements in postdiagnosis mortality and morbidity outcomes over the evaluated period. Respondents were provided with a fifth category, "cannot allocate," to account for postdiagnosis outcome gains resulting from other factors such as public health interventions. RESULTS: The conditions identified as having the greatest effect on morbidity and mortality since 1990 were breast cancer, ischemic heart disease, human immunodeficiency virus infection, diabetes, unipolar depression, chronic obstructive pulmonary disease, cerebrovascular disease, and lung cancer. After excluding other factors, physicians specializing in these conditions, with a mean of 21.4 years in practice, considered pharmaceuticals and biopharmaceuticals as having the greatest postdiagnosis effect across all 8 conditions, with $56 \%$ of outcome gains attributed to this innovation category. Diagnostics was the second biggest contributor at $20 \%$.

CONCLUSIONS: Physician perceptions indicated that attention should be paid to value assessments of innovative diagnostics, devices, and surgical procedures, as well as to pharmaceuticals and biopharmaceuticals, before goals for allocating health care expenditures among the different innovations are determined.

J Manag Care Spec Pharm. 2019;25(1):66-71

Copyright $\odot 2019$, Academy of Managed Care Pharmacy. All rights reserved.

\section{What is already known about this subject}

Medical technology innovations, such as diagnostics, medical devices, procedures, and prescription drugs, have increased life expectancy and quality of life after diagnosis for many diseases. Previous research has demonstrated that investment in medical technology innovation has led to increased health outcomes, and the value of investment in specific innovations, particularly in new pharmaceuticals or biopharmaceuticals, has frequently been shown through evaluations of the associated health outcomes and costs.

- Value assessments for innovative medical technologies and interventions are an important consideration in current debates on access and affordability of health care in the United States.

\section{What this study adds}

- This study recorded practicing physician impressions of the historical effect of 4 categories of postdiagnosis innovations in medical technology on patient outcomes within the 8 health conditions that have the largest effect on health in the United States. Findings indicated that, of the 4 categories, pharmaceutical and biopharmaceutical products were the most important postdiagnosis medical technology contributor to improvements in outcomes for the top causes of mortality and morbidity since 1990. These findings suggest that value assessments should be applied broadly to all medical technologies to ensure the best return on investment in health care services.

A ccording to the Centers for Disease Control and Prevention's "Chart Book on Long-Term Trends in Health, 2016," "advances in medical technology, including diagnostic imaging technologies, procedures, medical devices, and new prescription drugs have extended and improved the quality of countless lives." These advances in medical technology have improved population health in those at risk for a disease, as well as for those diagnosed with a disease. Medical technologies for disease prevention include vaccination and drug prophylaxis. Screening using diagnostic imaging or other procedures can identify diseases at earlier and more treatable stages. Diagnostic imaging, devices, surgical procedures, and new prescription drugs can increase cure rates, slow disease progression, and/or reduce symptoms after diagnosis. 
These medical technology-related reductions in disease incidence, changes in disease stage at diagnosis, and improvements in disease outcomes after diagnosis have been accompanied by rising health care costs. In the United States, some researchers have attempted to relate these increases in health care expenditures for prevention and treatment over time to related health improvements at an aggregate level to quantify the value of investment in health care services. For example, Cutler et al. (2000) sought to address how medical spending has translated to improvement in outcomes by analyzing life expectancy estimates and lifetime costs of medical care. ${ }^{2}$ They concluded that, although medical spending has increased at a fast pace for decades, the return on spending in the form of improvements in outcomes has also been high. ${ }^{2}$ Luce et al. (2006) took a multifaceted approach to estimating the return on investment in health care and concluded that the historical value of improved outcomes has outweighed the additional expenditures for the advances. ${ }^{3}$ In addition, Cutler and McClellan (2001) analyzed innovations in 5 specific conditions to further explore the balance of costs and benefits and found that spending as a whole is worth the increased cost. ${ }^{4}$

An assessment of value for different types of innovative medical technology interventions is an important consideration in current U.S. debates on access and affordability of health care. Whereas many published studies have focused on the value of new drugs because of the availability of clinical trial data, there is a growing movement in the United States to establish methodologies for estimating value that can be applied more broadly to all health care services. In response to the increased attention to costs and spending-particularly within the pharmaceutical, biopharmaceutical, diagnostics, and medical device sectors-several organizations, including the Institute for Clinical and Economic Review and medical professional societies such as the American Society for Clinical Oncology, have introduced frameworks to assess the value of all health care innovations and medical technologies. ${ }^{5}$

Value assessments are an appropriate way to make decisions regarding access to medical technologies to maximize health gains over time while ensuring affordability of U.S. health care. Such assessments should be performed for drugs (14.1\% of national health expenditures), ${ }^{6}$ as well as all other types of health care interventions, including screenings, behavioral counseling, diagnostics, devices, and surgical procedures (approximately $85.9 \%$ of spending on health care services) to ensure that decisions regarding access to different health care services or medical technologies will lead to the best health outcomes. To ensure the best health outcomes, estimates are needed to determine the effect of different types of medical technologies on health outcomes.

The purpose of this study was to present results from an exploratory analysis of the effect on health outcomes after diagnosis of different types of medical technologies based on a survey of practicing physicians. In this survey, physicians provided their perceptions of the relative effects on patient outcomes since 1990 of different types of postdiagnosis innovations in medical technologies within the 8 health conditions that have the largest effect on health in the United States.

\section{Methods}

National-level statistics were used to identify the top 8 conditions that continue to be responsible for the most mortality and morbidity within the United States since 1990 and the changes in morbidity and mortality for each condition since 1990 (Figure 1) ${ }^{7-9}$ Beginning in 1990 and continuing through 2014, the Centers for Disease Control and Prevention (CDC) and Global Burden of Disease databases included detailed information on diseases, which allowed for the identification and ranking of the top 8 conditions causing morbidity and mortality. The effects of the morbidity and mortality of these 8 conditions were based on the number of people with the conditions, as well as the effect of the condition on patient wellbeing and life expectancy after diagnosis. Based on the relative rankings of morbidity and mortality as defined by these national-level datasets, the top conditions were, in descending order, ischemic heart disease; cancer of the trachea, bronchus, or lung; breast cancer; human immunodeficiency virus (HIV); cerebrovascular disease; chronic obstructive pulmonary disease (COPD); unipolar depression; and diabetes. ${ }^{7-9}$

Between December 2016 and March 2017, surveys for each of the 8 conditions were developed and fielded to 158 U.S.based physicians, from whom 136 responses (86\% response rate), representing specialists in the 8 therapeutic areas, were obtained. Physicians were selected from a nationally representative physician panel of specialists currently practicing within the specialty, with at least 15 years of experience for each of the top conditions identified. The survey was submitted to the RTI International Institutional Review Board for approval, which determined that the scientific activity did not constitute research that involved human subjects as defined by the U.S. Code of Federal Regulations (45 CFR 46.102). A review of Centers for Medicare \& Medicaid Services (CMS) Open Payment data was also performed to assess representativeness of the physician sample and any potential conflicts of interest in relation to specialty means for payments.

The survey requested information regarding physician demographics, board certification, and practice setting and characteristics, as well as national-level data for the condition aligned with the physicians' respective specialties. A question then asked physicians to allocate 100 percentage points for all postdiagnosis improvements in mortality and morbidity of the condition within their specialties over the last 20 years among 4 categories of medical technology. These 4 categories were pharmaceuticals and biopharmaceuticals, devices, diagnostics, and surgical procedures. A fifth category, "cannot allocate," 


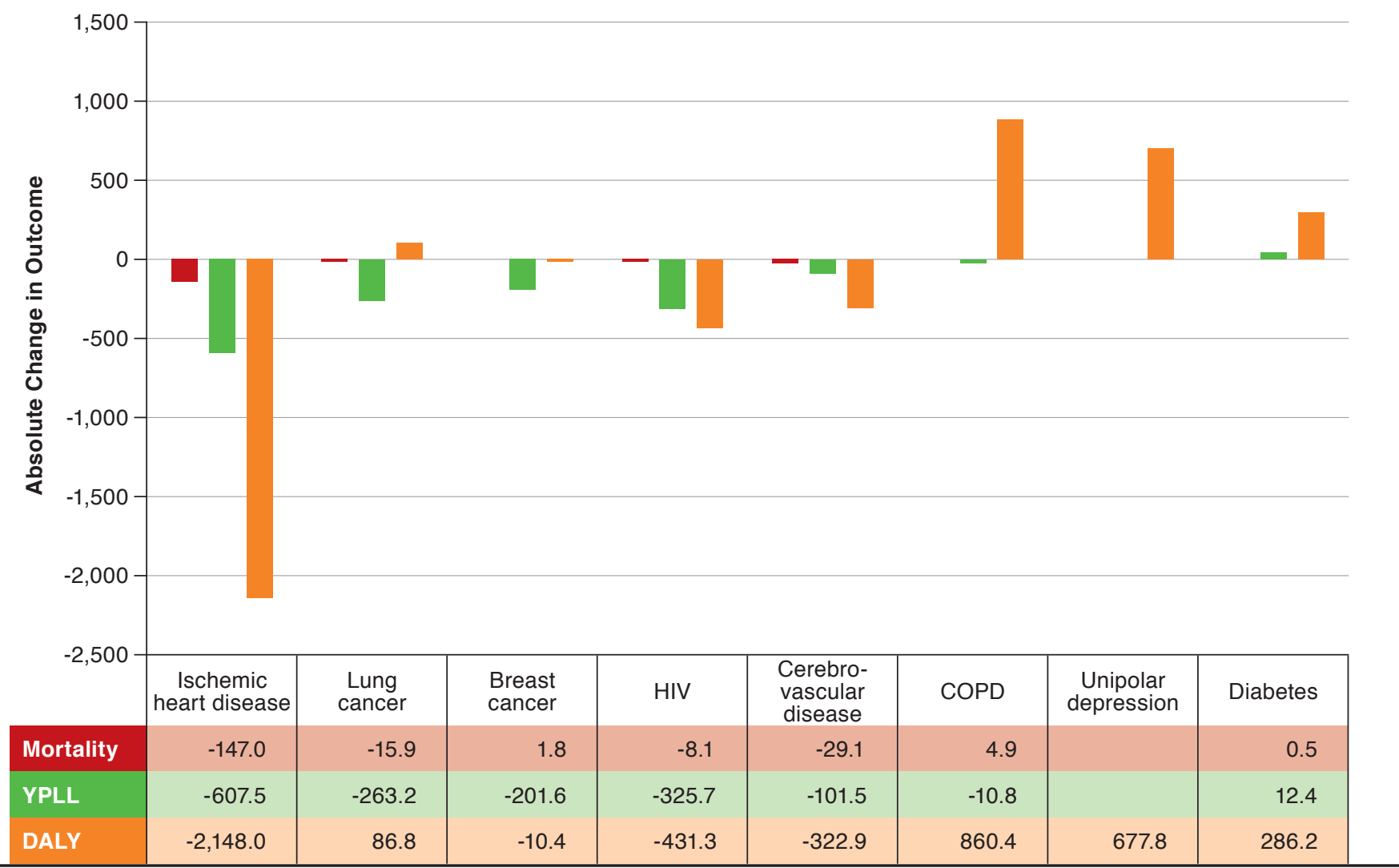

a Data from Centers for Disease Control and Prevention and the World Health Organization.7,8

$C O P D=$ chronic obstructive pulmonary disease; DALY=disability-adjusted life-year; HIV = human immunodeficiency virus; YPLL=years of potential life lost.

was also included as an option for participants to account for morbidity and mortality gains after diagnosis that resulted from other factors that might include secondary prevention and public health interventions. As an example, the following question was from the cerebrovascular disease survey: "Among the 4 categories of postdiagnosis innovations listed below, what percentage of the total effect on morbidity and mortality outcomes since 1990 do you feel each category of innovation accounts for? Please ensure that the percentages add up to $100 \% . "$

Unadjusted for prevalence, data on morbidity and mortality within 3 of the 8 conditions indicated higher morbidity and mortality rates since 1990 . These conditions were COPD, unipolar depression, and diabetes. Since estimates by the CDC included the effect of changes in the number of people with the disease, as well as changes in morbidity and mortality for those diagnosed with the disease, respondents to the unipolar depression survey, for example, were asked: "Based on these outcomes data that have only been adjusted for age, one may conclude that there have not been clinical improvements in unipolar depression since 1990. Do you think there have been improvements in clinical outcomes in unipolar depressive disorders since 1990?" The response categories were "yes" and "no." Physicians who responded "no" were not asked to complete the allocation exercise and the remainder of the survey.

Descriptive analysis of physician responses to the allocation exercise was performed as follows. First, we calculated the percentage allocations of effect to the different response categories for each disease and for each physician using 100 possible points as the denominator and the physician's assignment of points to each response category as the numerator. To focus on the effect of medical technology for each physician, we then calculated adjusted percentage allocations of effects for the 4 medical technology categories after removing the number of percentage points allocated to the "cannot allocate" response category from the denominator for each disease. Next, the mean percentage allocations of effect to each of the 4 medical technology categories were estimated for each disease by pooling data from all the physicians responding for each disease. Standard 


\section{FIGURE 2 Allocation of Relative Importance of 4 Innovation Categories to Changes in Health Outcomes ${ }^{\mathrm{a}}$}

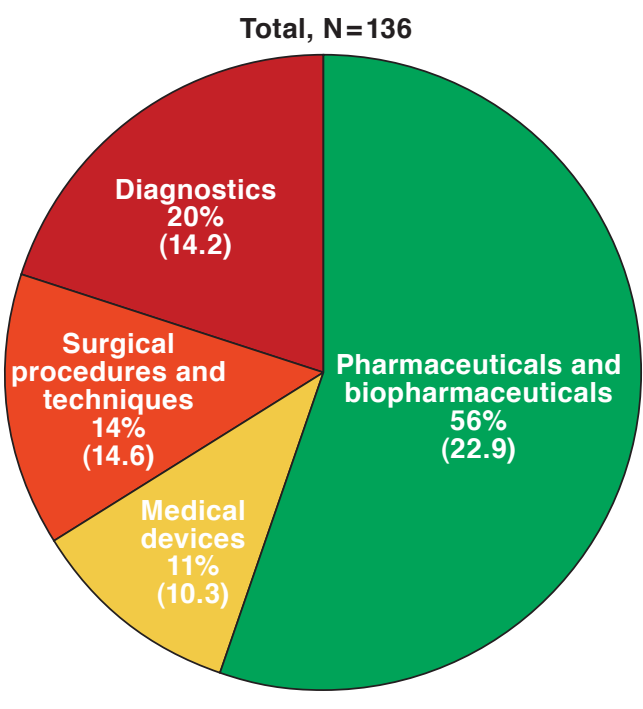

Therapy by Area, \% (SD)

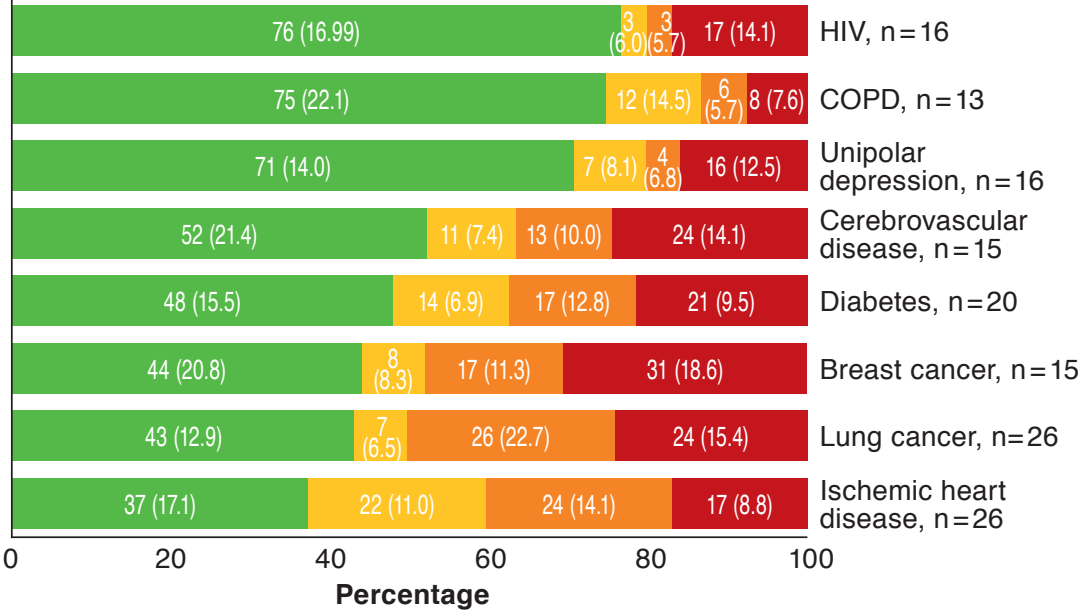

a Allocation percentages are based on 100 possible points assigned by physicians for all postdiagnosis improvements in mortality and morbidity of the condition within their respective specialties over the last 20 years among 4 categories of interventions: pharmaceuticals and biopharmaceuticals, devices, diagnostics, and surgical procedures. A fifth category, "cannot allocate," was included in the survey but these results represent the allocation percentages only for the 4 medical technology categories. Mean (SD) percentage responses to "cannot allocate" question were the following: breast cancer 2.67 (5.63), lung cancer 2.67 (4.95), cerebrovascular disease 1.33 (3.52), chronic lower respiratory disease 1.54 (5.55), HIV 0.31 (1.25), unipolar depression 1.25 (3.42), diabetes 13.7 (30.38), ischemic heart disease 2.31 (5.87), and pooled data 3.52 (12.89). $\mathrm{COPD}=$ chronic obstructive pulmonary disease; HIV=human immunodeficiency virus; $S D=$ standard deviation.

deviations were also estimated for the percentage allocations. We also pooled the data on the percentage allocations of effect for each of the 4 interventions for the 8 diseases to obtain an overall estimate of the mean percentage allocations of effect and standard deviations for the 4 medical technology categories. Finally, we tested for significant differences between the medical technology category receiving the highest allocation and each of the other medical technology categories in the pooled data.

\section{Results}

Of the 136 physicians who completed the survey, the mean number of years in practice was 21 years, with 94 (69\%) physicians having 20 or more years in practice. Most of the physicians surveyed $(n=118,87 \%)$ were board certified in their respective fields, and only 1 physician $(0.7 \%)$ had ever been employed by a pharmaceutical or biotech company. Of the 136 physicians completing the survey, 126 provided Open Payment information for 2016. This information indicated that 55 (44\%) physicians reported total general payments above specialty means from a dollar perspective. Because of responses indicating a perception of no improvement in postdiagnosis outcomes, 10 of 30 participants in the diabetes survey, 4 of 20 participants in the unipolar depression survey, and 8 of 21 participants in the COPD survey were not asked to complete the allocation questions.
Findings from the allocation portion of the survey (Figure 2) indicated that pharmaceuticals and biopharmaceuticals were perceived by the physicians to account for the greatest effect on mortality and morbidity across the 8 top conditions since 1990. The amount allocated to the "cannot allocate" category was low and less than a mean value of $4 \%$ for all responding physicians in all conditions other than diabetes (Figure 2). Two respondents to the diabetes survey allocated more than $75 \%$ of benefits to the "cannot allocate" category, driving the mean "cannot allocate" level to $13.7 \%$. After removal of this category across all conditions, physicians attributed 56\% (95\% confidence interval $[\mathrm{CI}]=52.03 \%-59.84 \%$ ) of patient outcomes gained to innovations in pharmaceuticals and biopharmaceuticals, followed by diagnostics at 20\% (95\% $\mathrm{CI}=17.26 \%-22.13 \%)$. Surgical procedures and techniques and medical devices had a lesser effect on mortality and morbidity, accounting for $14 \%$ (95\% CI $=11.19 \%-16.16 \%)$ and $11 \%(95 \%$ $\mathrm{CI}=8.94 \%-12.45 \%)$ of outcomes gained, respectively. In addition to being the single biggest overall contributor to outcome gains, pharmaceuticals and biopharmaceuticals was the biggest contributor in each of the 8 identified conditions (Figure 2). The results across all 8 conditions were similar, with diagnostics ranked as the second-largest contributor to outcomes gains in all conditions, except lung cancer, ischemic heart disease, and COPD, where the second-largest contributors were 
surgical procedures (lung cancer and ischemic heart disease) and medical devices (COPD).

\section{Discussion}

A sample of U.S. physicians selected from a nationally representative market research panel provided their perceptions of the effect of different categories of medical technology innovations on outcomes for patients diagnosed with the most debilitating health conditions in the United States. Findings indicated that pharmaceutical and biopharmaceutical products were the most important postdiagnosis medical technology contributor to improvements in outcomes for the top causes of mortality and morbidity since 1990. These findings provide an important perspective to the growing debate on the value of medical technology innovation and help illustrate that the physician-perceived benefits provided by pharmaceutical and biopharmaceutical innovations are substantial compared with other postdiagnosis innovations.

Physician perceptions regarding the historical effect of pharmaceuticals align with objective measures during the period studied within many of the top conditions identified. For example, the age-adjusted death rate per 100,000 for patients with HIV was 10.2 in 1990, declining to 2.1 in $2013 .{ }^{9}$ Further analysis indicated that, in addition to public health efforts, much of this progress can be attributed to advancements in pharmaceuticals, such as highly active combination antiviral agents. In addition, for cerebrovascular disease, the age-adjusted death rate per 100,000 patients was 65.3 in 1990, declining to 36.2 in $2013,{ }^{9}$ with physicians indicating that pharmaceutical innovations such as statins, novel anticoagulants, and tissue plasminogen activators were important contributors to improved outcomes.

Findings from this study highlight important policy implications relevant to determining access to innovative medical technology. Spending limits or caps on individual health care sectors may appear to be an adequate solution to health care costs concerns, but such measures should consider which areas of innovation bring the greatest value to patients and the health care system as a whole. These findings suggest that one way to assign scarce health care resources among different health care services could be to allocate them based on the benefit that the resources bring to patient outcomes. To do this for new medical technologies would require value-based assessments of all diagnostic, device, and surgical interventions, using methods similar to those commonly used for new drugs. In addition, public dialogue regarding the level of health care spending in general requires appropriate consideration of the population returns on investment in health care compared with other uses of public or private funds.

\section{Limitations}

This study has several limitations. First, we used subjective assignments of importance for the different innovations on patient outcomes. However, the survey relied on physician participants who averaged more than 20 years of clinical experience and had first-hand knowledge of how patient care and outcomes have been affected by innovations. The survey respondents also were unaware of the study hypothesis. In addition, the respondents provided independent evaluations without interacting with each other.

Second, although national-level statistics from the CDC and Global Burden of Disease databases were used to identify top conditions and define morbidity and mortality estimates for each condition, this approach may not have incorporated sufficient granularity within certain conditions. For example, breast cancer and lung cancer, both included in the top conditions, may have required more detailed considerations for stages of disease and lines of treatment to accurately assess the effect of postdiagnosis innovations on outcomes within the condition.

Finally, survey respondents were selected from a nationally representative physician panel of specialists who indicated willingness to participate in survey research. Therefore, the sample may have introduced potential conflicts of interest associated with the financial involvement of the physicians with pharmaceutical and biopharmaceutical manufacturers or other business relationships with health care organizations. However, results from the analysis of CMS Open Payment data indicated that the physician sample was representative of national-level norms for specialist colleagues related to means of payments and number of transactions with health care organizations.

\section{Conclusions}

Findings from this study indicate that, according to physician perception, over the last 20 years the measure of improvement in health outcomes after diagnosis for the 8 most debilitating diseases attributable to innovations in diagnostics, medical devices, and surgery may be less than that attributable to new drugs. Physician perception indicates that attention should be paid to value assessments of innovative diagnostics, devices, and surgical procedures, as well as to pharmaceuticals and biopharmaceuticals, before goals for allocating health care expenditures among the different medical technology innovations are determined. 


\section{Authors}

DAVID E. WAMBLE, PhD, RTI Health Solutions, Research Triangle Park, North Carolina. MICHAEL CIARAMETARO, MBA, and ROBERT DUBOIS, MD, PhD, National Pharmaceutical Council, Washington, DC.

AUTHOR CORRESPONDENCE: David E. Wamble, PhD, Director, Health Economics, RTI Health Solutions, 200 Park Offices Dr., Research Triangle Park, NC 27709. Tel.: 919.541.6646;

E-mail:dwamble@rti.org.

\section{DISCLOSURES}

Funding for this study was provided by the National Pharmaceutical Council, a health policy research group that receives its funding from biopharmaceutical manufacturers. Wamble is employed by RTI Health Solutions, which received funding from the National Pharmaceutical Council to conduct this research. Ciarametaro and Dubois are employed by the National Pharmaceutical Council.

\section{REFERENCES}

1. National Center for Health Statistics. Health, United States, 2016: With Chartbook on Long-Term Trends in Health. Hyattsville, MD. 2017. Available at: https://www.cdc.gov/nchs/data/hus/husl6.pdf. Accessed May 31, 2018.
2. Cutler DM, Rosen AB, Vijan S. The value of medical spending in the United States, 1960-2000. N Engl J Med. 2006;355(9):920-27.

3. Luce BR, Mauskopf J, Sloan FA, Ostermann J, Paramore C. The return on investment in health care: from 1980 to 2000. Value Health. 2006;9(3):146-56.

4. Cutler DM, McClellan M. Is technological change in medicine worth it? Health Aff (Millwood). 2001;20(5):11-29.

5. Neumann PJ, Cohen JT. Measuring the value of prescription drugs. N Engl J Med. 2015;373(27):2595-97.

6. Roehrig C. A ten year projection of the prescription drug share of national health expenditures including non-retail. Altarum Institute. Updated May 2017. Available at: https://altarum.org/sites/default/files/uploaded-publication-files/Non-Retail\%20Rx\%20Forecast\%20Data\%20Brief\%20with\%20 Addendum\%20May\%202017.pdf. Accessed May 31, 2018.

7. World Health Organization. Global health estimates (GHE). Available at: http:// www.who.int/healthinfo/global_burden_disease/en/. Accessed May 31, 2018.

8. World Health Organization. Disease burden and mortality estimates for 2000-2016. November 2013. Available at: http://www.who.int/healthinfo/ global_burden_disease/estimates/en/index2.html. Accessed May 31, 2018.

9. National Center for Health Statistics. Health, United States, 2015: With Special Feature on Racial and Ethnic Health Disparities. Hyattsville, MD. 2015. Available at: www.cdc.gov/nchs/data/hus/hus15.pdf. Accessed May 31, 2018. 